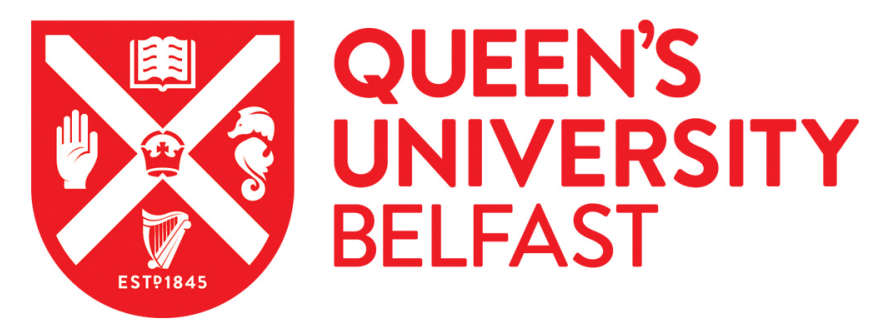

\title{
Nanodomain Patterns in Ultra-Tetragonal Lead Titanate (PbTiO3)
}

Kumar, A., Guy, J., Zhang, L., Chen, J., Gregg, M., \& Scott, J. (2020). Nanodomain Patterns in Ultra-Tetragonal Lead Titanate (PbTiO3). Applied Physics Letters, 116, [182903]. https://doi.org/10.1063/5.0007148

Published in:

Applied Physics Letters

Document Version:

Peer reviewed version

Queen's University Belfast - Research Portal:

Link to publication record in Queen's University Belfast Research Portal

Publisher rights

Copyright 2020 AIP. This work is made available online in accordance with the publisher's policies. Please refer to any applicable terms of use of the publisher.

\section{General rights}

Copyright for the publications made accessible via the Queen's University Belfast Research Portal is retained by the author(s) and / or other copyright owners and it is a condition of accessing these publications that users recognise and abide by the legal requirements associated with these rights.

Take down policy

The Research Portal is Queen's institutional repository that provides access to Queen's research output. Every effort has been made to ensure that content in the Research Portal does not infringe any person's rights, or applicable UK laws. If you discover content in the Research Portal that you believe breaches copyright or violates any law, please contact openaccess@qub.ac.uk. 


\section{Nanodomain Patterns in Ultra-Tetragonal Lead Titanate $\left(\mathrm{PbTiO}_{3}\right)$}

Amit Kumar, ${ }^{1,{ }^{\star}}$ Joseph G. M. Guy, ${ }^{1}$ Linxing Zhang, ${ }^{2}$ Jun Chen, ${ }^{2}$ J. Marty Gregg,,${ }^{1}$ and James F. Scott ${ }^{3}$

${ }^{1}$ School of Mathematics and Physics, Queen's University Belfast, Belfast, BT7 1NN, UK ${ }^{2}$ Department of Physical Chemistry, University of Science and Technology Beijing, Beijing 100083, China

${ }^{3}$ School of Chemistry and Physics, St. Andrews University, St. Andrews, Fife KY16 9AJ, UK *Address correspondence to: a.kumar@qub.ac.uk

\section{Abstract:}

Very recently, the discovery of ultra-tetragonal $\mathrm{PbTiO}_{3}$ thin films was reported [L. Zhang et al., Science, 361, 494 (2018)], in which the switchable out-of-plane polarization was seen to be almost twice that of any previously known ferroelectric. To understand more about this system and to explore features that might contribute to these remarkable functional properties, we have mapped the polarisation microstructure on the nanoscale using piezoresponse force microscopy (PFM) (scan sizes less than $700 \mathrm{~nm}$ ). Our study reveals an extremely fine-scale pattern, with individual domains typically $20-50 \mathrm{~nm}$ in size, all of which exhibit both out-of-plane and in-plane polarisation components. In-plane vector PFM polarisation maps show strongly charged quadrant-lattice domain arrays with four-fold domain wall junctions (vertices). The existence of four-fold vertices in ferroelectrics is rare but can be explained via the 'AshkinTeller' model, in which very large interfacial lattice mismatch strains and local electric fields play a role. This ultra-tetragonal ferroelectric system was expected to contain straightforward c-axis out-of-plane $180^{\circ}$ domains, but instead shows an extremely rich nanoscale domain tiling pattern; these unusual nanodomains may be important in facilitating the extremely high switchable polarisation values previously reported. 
Domain wall vertices (or meeting points) are established and ubiquitous features in ferroelectric microstructures ${ }^{1,2,3,4}$, but historically they have not been considered as being particularly important. This view is now changing: from an applied point of view, domain wall vertices have already been seen to act as $1 \mathrm{D}$ electrically conducting channels (in $\mathrm{BiFeO}_{3}$ thin films ${ }^{5}$ ); in addition, in some rare-earth manganite systems $s^{6,7,8}, 1 \mathrm{D}$-n junctions should form at six-fold vertices, where both conducting head-to-head (n-type) and tail-to-tail ( $p$-type) domain walls meet; new forms of domain wall Josephson junctions have even been proposed, in systems such as $\mathrm{WO}_{3-x}$ where domain wall superconductivity has already been established. ${ }^{9},{ }^{10}$ From a fundamental point of view, the energetics associated with domain wall vertex formation, dynamics ${ }^{11}$ and the underpinning physics associated with their topological form are interesting and relatively unexplored; basic ideas are certainly much less well developed than they would be for domain walls, for example.

A phenomenological description by Srolovitz and Scott ${ }^{12}$, based on Potts and clock models, provides some theoretical basis upon which different vertex configurations may be rationalised: it focuses on whether 4-fold " $X$ " vertices or adjacent pairs of 3-fold " $Y$ " vertices are stable and the mechanisms by which vertex coalescence or separation might occur. According to the model, a four-state Potts model is required to describe the domain wall vertices in either of the two cases. However, to explain pairs of adjacent $Y$-shaped three-fold vertices, the Potts model involves scalars only (a simple extension of an Ising model). In contrast, a vector Potts model ("clock model") is required to describe the stable existence of four-fold vertices. In general, a given ferroelectric can exhibit three-fold or four-fold vertices, but not both. It is also useful to point out that the general question in nature of whether fourfold or three-fold vertices are stable is rather well examined. In two dimensions, Voronoi partitions (domain walls in the present context) subdivide space into four quarters only when the objects of interest are symmetrically placed on the vertices of a rectangle (nucleation sites at defects in the present example). Otherwise, any other configuration generates closely spaced pairs of three-fold vertices. This is a result of the growth of domains from initial nucleation sites to fill two-dimensional Euclidean space. ${ }^{13}$ The collapse of four-fold vertices into adjacent pairs of three-fold vertices is further considered by Opperman ${ }^{14}$, who also emphasizes qualitative changes in dynamics due to axial anisotropy. In general, static experimental observations in ferroelectrics suggest that pairs of separated three-fold vertices are more stable than more compact four-fold "core" vertex geometries. ${ }^{15,16,11}$ However, the converse may become true in thin films where the energetics may be dominated by the presence of a strong internal/external electric field. A very few systems illustrate both cases. This does not violate the Srolovitz-Scott model, but rather implies that the interaction parameter is anisotropic, with out-of-plane polarized domains satisfying a scalar Potts model and in-plane polarized domains satisfying a vector "clock" model. This behaviour has only 
been seen in lead strontium titanate (PST), ${ }^{17}$ where pairs of three-fold vertices are found and - "very rarely" - a few four-fold vertices, and $\mathrm{SrBi}_{2} \mathrm{Ta}_{2} \mathrm{O}_{9}(\mathrm{SBT})$ which exhibits both three-fold and four-fold vertex geometries.

It is within this context, where domain wall junctions should be explored more fully, that we report the existence of quadrant lattice domain arrays and four-fold in-plane domain vertices in the rather exotic ultra-tetragonal $\mathrm{PbTiO}_{3}$ thin-film ferroelectric system. It is well understood that negative pressure, induced by tensile strain, can result in dramatic changes in the structure and functionality of perovskite oxides, particularly in the case of ferroelectrics. ${ }^{18}$ One of the novel approaches to achieve this effect in thin epitaxial ferroelectric films is through the realisation of 'interphase strain', where two materials with similar crystal structures but different lattice parameters can be grown as a single epitaxial composite film such that their lattice parameters are matched. The isotropic strain generated in the individual phases, as a result of such lattice-matching, can induce negative pressure, thereby generating enhanced polarization, elevated Curie temperatures and large c/a ratios. This concept has recently been realised in $\mathrm{PbTiO}_{3}$ (PTO) - $\mathrm{PbO}$ epitaxial composite ferroelectric thin films, where the PTO phase exhibits giant switchable polarisation of approximately $236 \mu \mathrm{C} / \mathrm{cm}^{2}$ (nearly twice the record polarisation reported in any ferroelectric). ${ }^{19}$ The effect of such enhanced interphase strain on the functional aspects of this film have been examined macroscopically in the previous work. ${ }^{19}$ Commensurate with the dramatic enhancement in polarisation, it is expected that the enhanced strain would also influence the nanoscale domain structure of the $\mathrm{PbTiO}_{3}$ film, both in terms of periodicity as well as complexity of the domain patterns. Previous scanning probe investigations were undertaken on the mesoscale (several $\mu \mathrm{m}$ ) and did not reveal domain microstructures clearly. To address this, we investigate the nanoscale domain structure of this material using piezoresponse force microscopy (PFM). Surprisingly, dense domains (with domain diameters $20-50 \mathrm{~nm}$ in size) with both out-of-plane as well as in-plane polarisation components are observed. The in-plane polarisation mapping reveals quadrant lattice domain arrays, four-fold in-plane domain vertices and prolific occurrence of apparently charged domain walls in the same region.

The studied films were grown by radio-frequency magnetron sputtering using a $\mathrm{PbTiO}_{3}$ target, which was sintered at $1100^{\circ} \mathrm{C}$ for 2 hours. The samples were $50 \mathrm{~nm}$ thick and grown on $0.7 \mathrm{wt} \% \mathrm{Nb}$-doped $\mathrm{SrTiO}_{3}$ substrates. Details of the growth conditions have been reported elsewhere. ${ }^{19}$ The PFM measurements were undertaken on an Oxford Instruments MFPInfinity Atomic force microscope. The crystal structure of the films was investigated using $x-$ ray diffraction (XRD), which was performed on the diffractometer (PW3040/60, PANalytical, Holland) with $\mathrm{Cu} \mathrm{K}_{a}$ radiation. Single-frequency piezoresponse force microscopy (PFM) has 
been employed to investigate the nanometre scale domain structure in the studied strained PTO film. The out-of-plane X-ray investigation on these films, grown on (100) STO substrates, suggests lattice parameters consistent with the $\mathrm{PT}(\mathrm{I})$ phase previously reported by Zhang et al., thus indicating large $c$ values and consequently a large c/a ratio (as shown in Fig. 1(a). The reciprocal space map for the 103 Bragg peak of the studied film has been provided in supplementary information (Fig. S1 in supplementary information); it demonstrates that the inplane lattice parameter is the same as that of the substrate, and that the PTO film is hence demonstrating a super-tetragonal unit cell, commensurate with previous work. ${ }^{19}$ In this context, the out-of-plane vertical piezoresponse of the sample was first examined by applying 4V AC to a metal-coated PPP-EFM Nanosensors tip with a tip-surface contact resonance at $330 \mathrm{kHz}$ (the chosen frequency was close to the contact resonance and bias value was chosen for best contrast). As shown in Fig. 1(b), the surface of the film is quite smooth (roughness $<2 \mathrm{~nm}$ ) but shows some contrast in terms of granularity. The vertical phase and amplitude maps (Fig. 1(c) and $1(d)$ ) show evidence of up and down domains on a very fine scale, where the average size of the domains ranges from $50-80 \mathrm{~nm}$.

Mapping on finer length scales (550 nm x $550 \mathrm{~nm}$ ), the out-of-plane PFM contrast becomes even clearer, as shown in Fig. 2(a-c). Surprisingly, good in-plane contrast in lateral PFM measurements was also observed for exactly the same region (see matching topographies in Fig. 2(a,d,g)). These lateral PFM measurements were undertaken by applying $4 \mathrm{~V}$ ac with frequency closer to the lateral tip-surface resonance $(650 \mathrm{kHz})$ and the measurements were conducted in two perpendicular tip-sample orientations (sample rotated with respect to the tip). In both orientations, the amplitude and phase images (Figs. 2(e-f) and $2(\mathrm{~h}-\mathrm{i}))$ are well resolved and reveal domain widths on the scale of $\sim 50 \mathrm{~nm}$. Overlays of vertical and lateral PFM responses onto the topography of the film are provided in supplementary information (Fig. S2 in supplementary information). Lateral PFM signals (amplitude and phase) show correlations across many grains in strips - perhaps indicative of grain-to-grain polarisation coupling. Vertical PFM response shows correlations with the undulations ( $2 \mathrm{~nm})$ in topography. Any concerns about cross-talk were, however, allayed by nearly identical VPFM scans observed at different scan angles (Fig. S3 in supplementary information) as well as observations of tip-induced switching which show domain growth and relaxation, unrelated to the sample topography (Figs. S4 and S5 in supplementary information). Overall, PFM data reveals that the domains in this epitaxial composite film have both in-plane as well as out-ofplane components, prompting a more careful analysis of the domain microstructure. Ricinshi et al. have shown that strain can produce in-plane and out of plane domains in the same surface ${ }^{20}$ depending upon sample thickness and strain. Hence, the observation of both inplane and out-of-plane polarisation components in this film is not entirely unexpected. 
The two in-plane lateral PFM images obtained upon rotating the sample with respect to the tip by $90^{\circ}$ were merged to obtain the 'vector' map of in-plane polarisation observed in the sample. The phase images were both corrected to remove phase wrapping and binarised before merging them at every pixel (as shown in Fig. 3). The net in-plane polarisation map reveals quadrant lattice domain arrays with periodicities of around $100 \mathrm{~nm}$. In these quadrant arrays, careful analysis of the polar directions reveals alternating convergence and divergence at vertices (centred-type patterns). As a result, the domain configuration suggests surprising but prolific existence of apparently charged walls. It should be noted that such measurements have been made carefully on two different sets of films and found to produce the same form of microstructure. To investigate the electronic properties of the charged walls, conduction mapping was undertaken by applying $+/-12 \mathrm{~V}$ to the bottom electrode, but no observable current was obtained across the sample; this does not necessarily imply that the charged domain walls are electrically insulating, as the $\mathrm{PbO}$ lenses in the microstructure might prevent conduction pathway percolation through the entire film thickness.

Importantly, most of the vertices observed in this combined in-plane polarisation map are four-fold. These four-fold vertices are expected by Srolovitz and Scott ${ }^{12}$ under conditions identified in the Ashkin-Teller model, named after the scientists who first published the relevant equivalent interaction ideas. ${ }^{21}$ It is still studied in the case of a large externally applied field, ${ }^{22}$ which may be the case here, given the large interfacial lattice mismatch strain and preponderance of charged polarisation patterns. We emphasize that in the absence of such a large field, three-fold vertices are generally dominant. In the composite lateral map shown in Figure 3 however, we observe very few three-fold vertices. Moreover, it is evident from Fig. 2 that the film has co-existing in-plane and out-of-plane polarisation components. By merging the in-plane vector map obtained in Fig. 3 with Fig. 2(c) (phase cleaned and binarised), we obtain a $3 \mathrm{D}$ visualisation of the domain microstructure which presents as an even more complex domain pattern shown in Fig. 4. The visualisation suggests that the 'apparent' charged walls (discussed in Fig. 3 ) retain their charged nature even after the inclusion of outof-plane domain orientation information.

In light of the observation of the dominance of four-fold vertices in the in-plane domain maps, we can discuss another possible physical reason for why the out-of-plane domains satisfy a scalar Potts model and the in-plane domains conform to the vector Potts prediction. It is worth pointing out that these domains are not topologically equivalent, with out-of-plane polarizations allowed to be only up or down while the in-plane domains are allowed to be oriented along four directions. In two dimensions, closely spaced pairs of three-fold vertices is the preferred case (for Voronoi partitions and domain walls) and Weaire and Aste describe this ${ }^{23,24}$ as a two-dimensional law. However, in three dimensions the opposite is true, and four- 
fold vertices are stable. Our films ( $\sim 0 \mathrm{~nm}$ thick and roughly equal to the size of lateral domains) represent a pseudo two-dimensional system, and thus could behave like a threedimensional system. Consequently, the change of vertex geometry from four-fold for in-plane polarisation to three-fold for out-of-plane polarization may derive simply from the effective dimensionality in the two cases. For the studied system, we prefer the strong axial field model ${ }^{21}$ as an explanation (Ashkin-Teller model) for our observed four-fold in-plane vertices, but include the Voronoi discussion for completeness. It is also interesting to note that the observed domain walls are charged, meaning polar discontinuities could create strong electric field which could drive the existence of four-fold vertices as seen in in-plane polarisation maps.

An unusual feature of the maps presented in Fig. 4 is that eight domain orientations are implied (Fig. 4(c)), as opposed to the six expected for the tetragonal and supertetragonal states. While there may be a possibility that the PTO has adopted another symmetry (a pseudo-rhombohedral state for example), we suspect a more mundane origin: eight apparent domain variants would appear under vector PFM analysis if the microstructure were composed of finescale $180^{\circ}$ domains (with polar axes pointing into and out of the plane of the film), as long as the physical orientations of unit cells could meander slightly across the film surface. Such mosaicity, due to a range of very slight crystallographic misorientations or lattice bending, could readily result from coherent cloaking of the subsurface $\mathrm{PbO}$ lens structures, which are such a characteristic microstructural feature in these supertetragonal films. Moreover, mosaicity would be consistent with the slight curvature of the 103 Bragg reflection seen in reciprocal space maps (Fig. S1 of the supplementary information) and the absence of multiple diffraction spots and Bragg splitting normally seen in conventional ferroelastic domain microstructures.

In summary, detailed nanoscale mapping of polarisation variants in an 'interphasestrain' mediated composite epitaxial film of $\mathrm{PbTiO}_{3}$ reveals surprising co-existence of out-ofplane and in-plane polarisation components. The in-plane polarisation maps reveal the existence of four-fold vertices and quadrant lattice domain arrays on remarkably small scales (a few tens of nanometers). The existence of four-four vertices in ferroelectrics is relatively rare and can be explained via the 'Ashkin-Teller' model, applicable due to very large interfacial lattice mismatch strain and electric fields from charged dipole configurations.

See supplementary material for additional information regarding XRD studies, overlays of PFM images on topography and results of attempts to switch the ferroelectric domains in limited voltage range.

The authors acknowledge financial support from the Engineering and Physical Sciences Research Council (contract EP/P02453X/1 and EP/S037179/1), the US-Ireland Research and Development Partnership Programme, the Queen's University Belfast Central Research Infrastructure Fund (CRIF) and the Northern Ireland Department for the Economy. 
J.G.M.G and A.K. would also like to thank the Engineering and Physical Sciences Research Council for Ph.D. studentship funding. The data that support the findings of this study are available from the corresponding author upon reasonable request. We dedicate this work to Prof. James F. Scott (this study was primarily driven by his efforts), who was a well-respected colleague and a dear friend to all of us.

\section{References:}

G. Arlt and P. Sasko, J. Appl. Phys. 51, 4956 (1980).

N. A. Pertsev and G. Arlt, Ferroelectrics 132, 27 (1992).

I. MacLaren, L. A. Schmitt, H. Fuess, H. Kungl, and M. J. Hoffmann, J. Appl. Phys.

97, 094102 (2005).

P. Mokry and J. Fousek, J. Appl. Phys. 97, 114104 (2005).

N. Balke, B. Winchester, W. Ren, Y. H. Chu, A. N. Morozovska, E. A. Eliseev, M.

Huijben, R. K. Vasudevan, P. Maksymovych, J. Britson, S. Jesse, I. Kornev, R.

Ramesh, L. Bellaiche, L. Q. Chen, and S. V. Kalinin, Nat. Phys. 8, 81 (2012).

M. E. Holtz, K. Shayovalov, J. A. Mundy, C. S. Chang, Z. W. Yan, E. Bourret, D. A. Muller, D. Meier, and A. Cano, Nano Lett. 17, 5883 (2017).

J. A. Mundy, J. Schaab, Y. Kumagai, A. Cano, M. Stengel, I. P. Krug, D. M. Gottlob,

H. Doganay, M. E. Holtz, R. Held, Z. Yan, E. Bourret, C. M. Schneider, D. G. Schlom,

D. A. Muller, R. Ramesh, N. A. Spaldin, and D. Meier, Nat. Mater. 16, 622 (2017).

P. W. Turner, J. P. V. McConville, S. J. McCaftan, M. H. Campbell, J. Schaab, R. G.

P. McQuaid, A. Kumar, and J. M. Gregg, Nano Lett. 18, 6381 (2018).

A. Aird and E. K. H. Salje, J Phys.-Condens. Mat. 10, L377 (1998).

E. K. H. Salje, Domain Walls: From Fundamental Properties to Nanotechnology Concepts. (Oxford University Press, 2020).

R. G. P. McQuaid, A. Gruverman, J. F. Scott, and J. M. Gregg, Nano Lett. 14, 4230 (2014).

D. J. Srolovitz and J. F. Scott, Phys. Rev. B 34, 1815 (1986).

X. H. Zhu, J. M. Zhu, S. H. Zhou, Q. Li, Z. G. Liu, and N. B. Ming, Appl. Phys. Lett. 78, 799 (2001).

R. Oppermann, J. Phys. A: Math. Gen. 8, L43 (1975).

L. J. McGilly, A. Schilling, and J. M. Gregg, Nano Lett. 10, 4200 (2010).

L. W. Chang, V. Nagarajan, J. F. Scott, and J. M. Gregg, Nano Lett. 13, 2553 (2013).

O. Nesterov, PhD Thesis, University of Groningen, 2015.

S. Tinte, K. M. Rabe, and D. Vanderbilt, Phys. Rev. B 68, 144105 (2003).

L. X. Zhang, J. Chen, L. L. Fan, O. Dieguez, J. L. Cao, Z. Pan, Y. L. Wang, J. G.

Wang, M. Kim, S. Q. Deng, J. O. Wang, H. H. Wang, J. X. Deng, R. B. Yu, J. F.

Scott, and X. R. Xing, Science 361, 494 (2018).

D. Ricinschi, M. Noda, M. Okuyama, Y. Ishibashi, M. Iwata, and L. Mitoseriu, J. Kor.

Phys. Soc. 42, S1232 (2003).

J. Ashkin and E. Teller, Phys. Rev. 64, 178 (1943).

B. Berche, P. Butera, and L. N. Shchur, J. Phys. A: Math. Theor. 46, 095001 (2013).

D. Weaire and T. Aste, The pursuit of perfect packing. (CRC Press, Cleveland, 2000).

D. Weaire and Rivier N., Contemp. Phys 25, 59 (1984). 


\section{Figures}

(a)

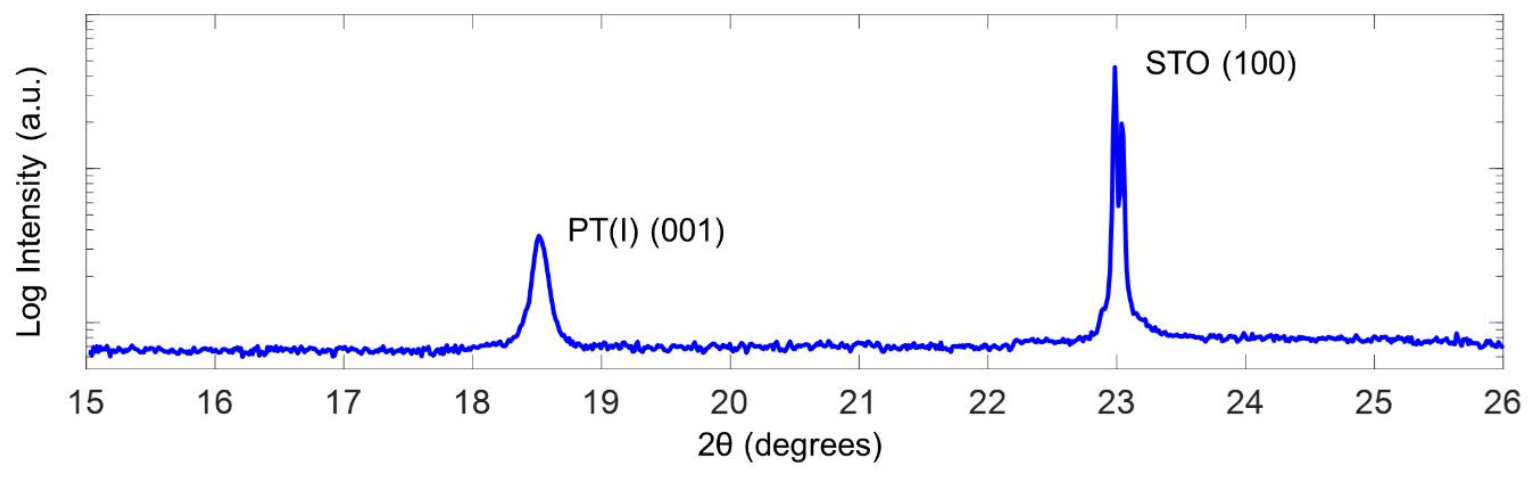

(b)

(c)

(d)

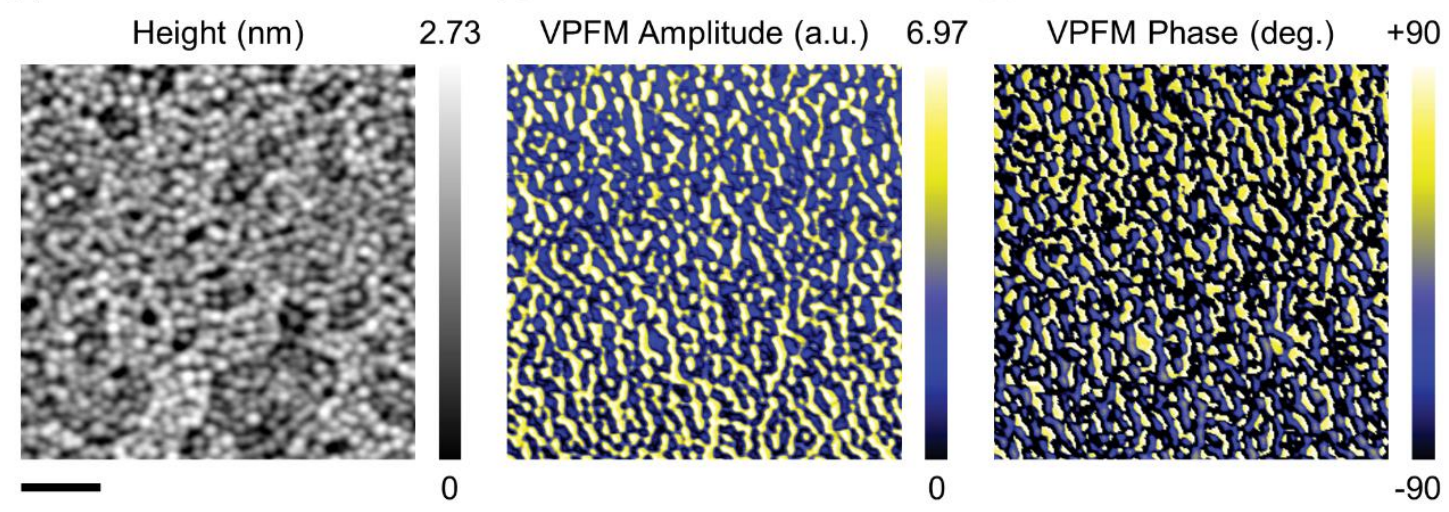

Figure 1: Ultra fine domain structure in super-tetragonal thin films (a) X-ray diffraction (XRD) data illustrating the out-of-plane (00I) peaks for the PT(1) film as described in reference [19]. (b) Nanoscale topography of a representative region of this film shows reasonably smooth contrast overall (RMS roughness $<1 \mathrm{~nm}$ ) but fine-scale granular features are obvious. Vertical piezoresponse force microscopy (VPFM) amplitude (c) and phase (d) for the same region show distinct polarisation states (up and down). It is notable that the domain structure is very fine: the typical domain width is around $50-70 \mathrm{~nm}$. The scale bar shown in (b) measures $390 \mathrm{~nm}$. 
(a)

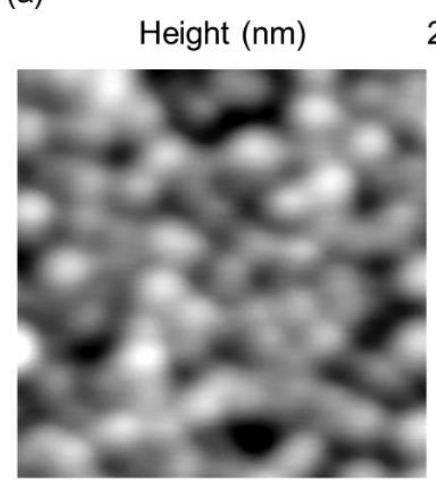

(d)

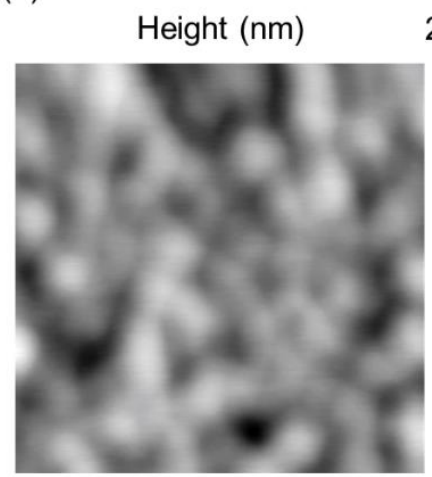

(g)

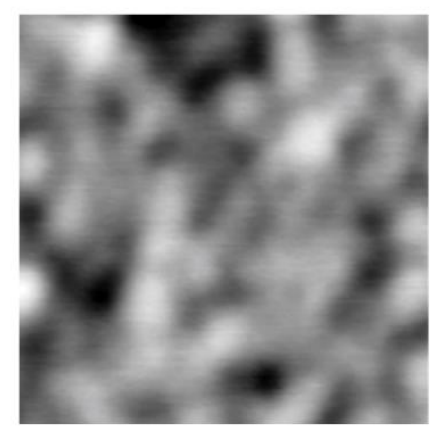

(b)

2.44 VPFM Amplitude (a.u.)

(c)

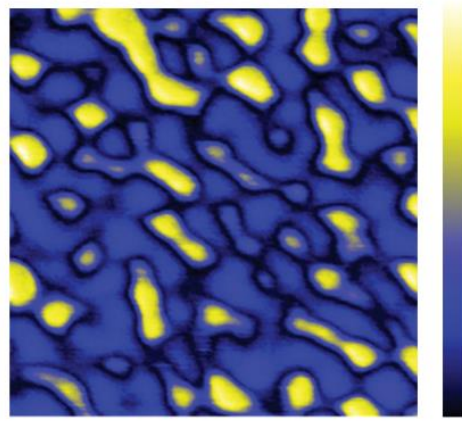

0

(e)

2.74

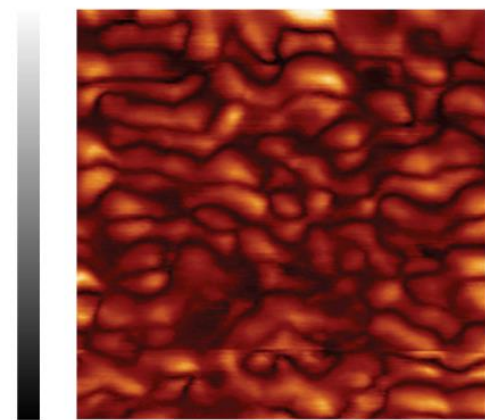

0

(h)

2.03 LPFM Amplitude (a.u)

8.23
0

(f)

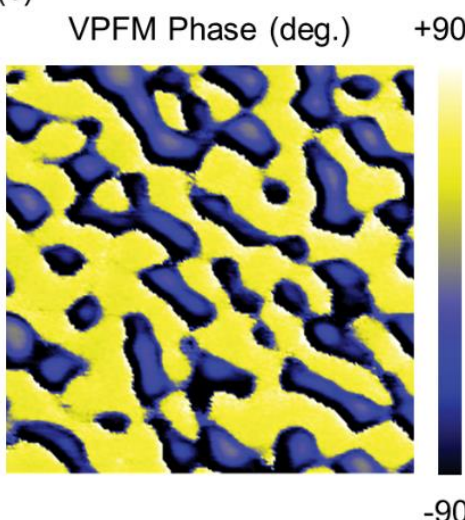

$-90$

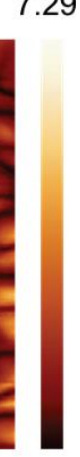

0

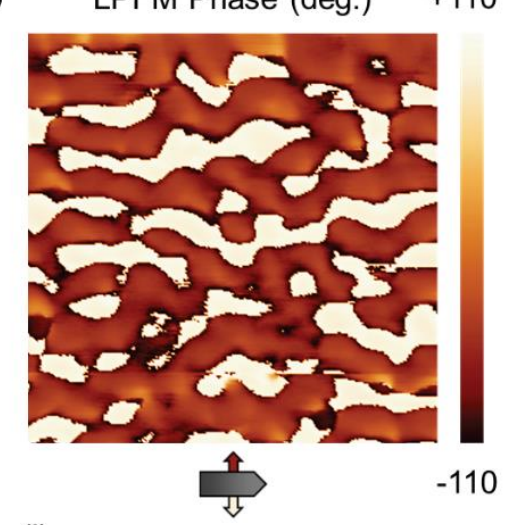

(i)

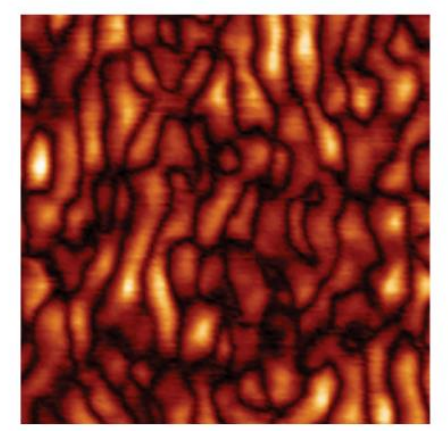

0

5.47

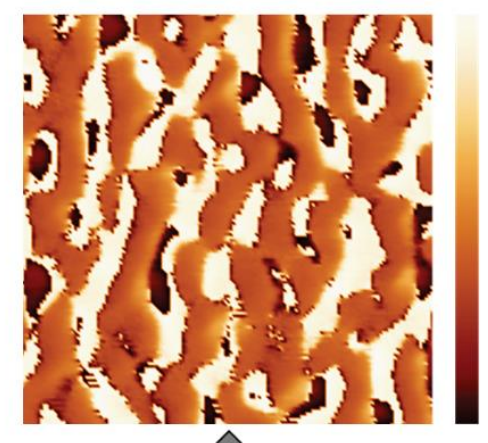

0

$-110$

Figure 2: Out-of-plane and in-plane piezoresponse force microscopy (PFM) in supertetragonal thin film (a-c) Topography (a), vertical PFM amplitude (b) and vertical PFM phase (c) from a region of the film surface; (d-f) Topography (d), lateral PFM amplitude (e) and lateral PFM phase ( $f$ ) for the same region as that shown in (a-c) (key topographic features are maintained); the cantilever orientation with respect to the sample is shown in panel (f) (as are the associated senses of the in-plane polar components); ( $g-i)$ Topography (g), lateral PFM amplitude (h) and phase (i) in the same region (again key topographic features remain the same) for the cantilever oriented perpendicular to that associated with panels (d-f). The scale bar measures $110 \mathrm{~nm}$. 
(a) Binarized LPFM Phase +1

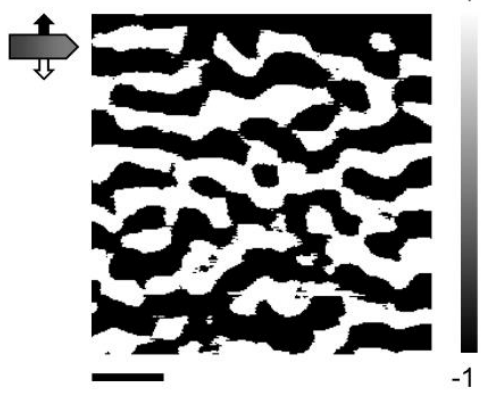

(b)

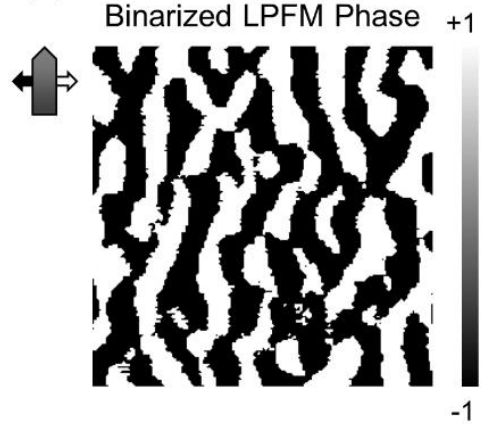

(c)

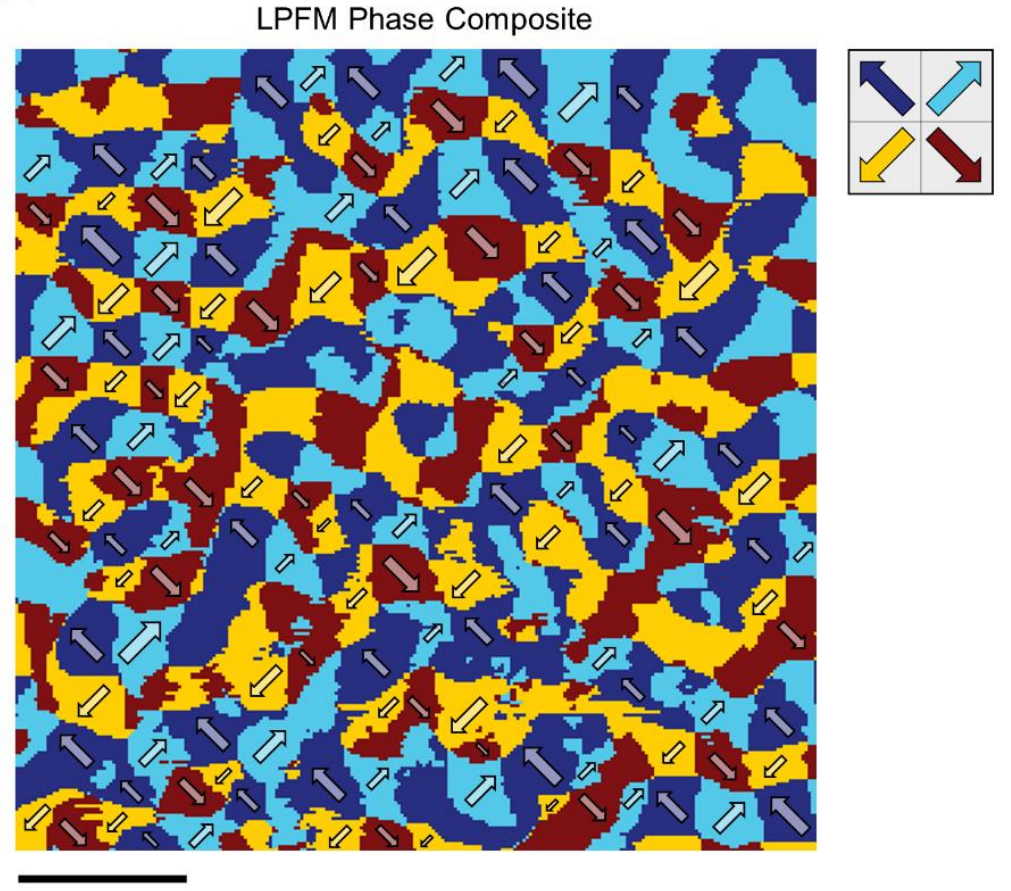

Figure 3: Collated lateral piezoresponse force microscopy (PFM) information shows inplane quadrant lattice domain arrays and the prolific existence of charged domain walls. Information from binarised in-plane domain maps (a) and (b) (from lateral PFM taken with the cantilever in two perpendicular orientations) is combined to create the vector map shown in (c). The colours in (c) indicate the direction of the in-plane components of the polar vector at each location. The scale bars in (a-c) measure $110 \mathrm{~nm}$. 
(a)

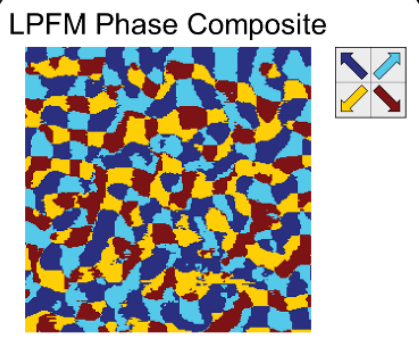

(b)

Binarized VPFM Phase +1

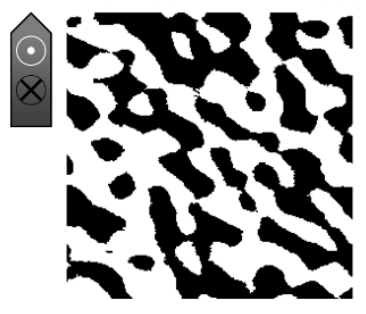

(c)

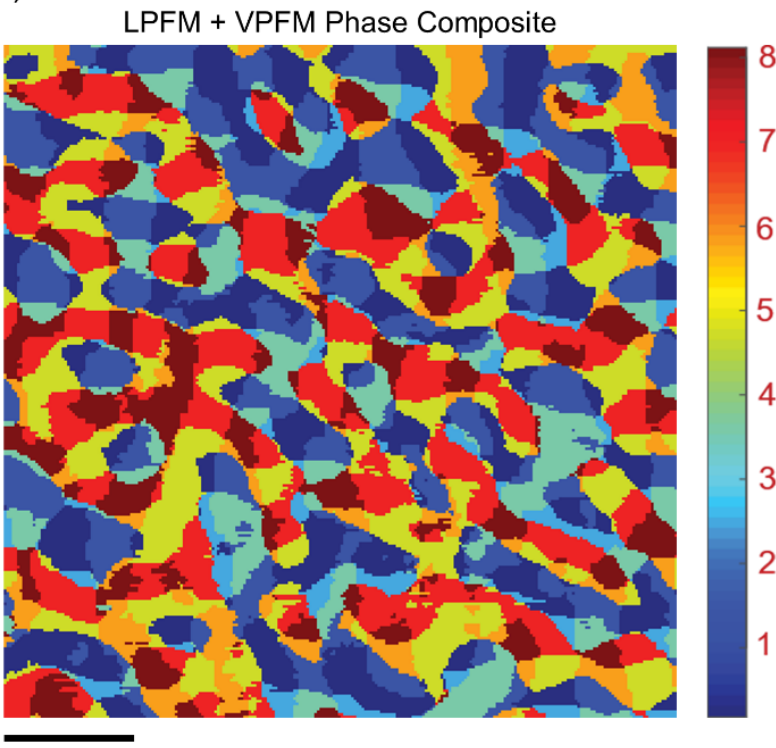

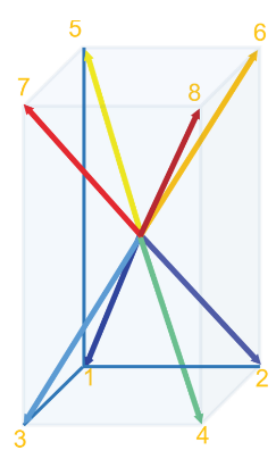

Figure 4: Inclusion of out-of-plane domain orientation information suggests an even more complex domain pattern. The merged in-plane map obtained in figure 3 is further merged with a binarized out-of-plane piezoresponse force microscopy (VPFM) map to obtain a threedimensonal visualisation of the polar variants at each pixel. The manner in which the colours map onto the sense of the resolved 3-D polar vector are made clear in the right-hand schematic. The scale bars in $(a, c)$ measure $110 \mathrm{~nm}$. 